\title{
Supplementary Information to" Many Body Response of Benzene at Monolayer MoS2: Van der Waals interactions and spectral broadening"
}

\author{
Alina Umerbekova ${ }^{1}$ and Michele Pavanello ${ }^{1}$ \\ ${ }^{1}$ Affiliation not available
}

May 5, 2020

\begin{abstract}
Models of surface enhancement of molecular electronic response properties are challenging for two reasons: (1) molecule-surface interactions require the simultaneous solution of the molecular and the surface dynamic response (a daunting task); (2) when solving for the electronic structure of the combined molecule+surface system, it is not trivial to single out the particular physical effects responsible for enhancement. To attack this problem, in this work we apply a formally exact decomposition of the system's response function into subsystem contributions by employing subsystem DFT which grants access to dynamic polarizabilities and optical spectra. In order to access information about the interactions between the subsystems, we extend a previously developed subsystem-based adiabatic connection fluctuation-dissipation theorem of DFT to separate the additive from the nonadditive correlation energy and identify the nonadditive correlation as the van der Waals interactions. As an example, we choose benzene adsorbed on monolayer $\mathrm{MoS}_{2}$. We isolate the contributions to the benzene's dynamic response arising from the interaction with the surface and for the first time, we evaluate the enhancements to the effective $\mathrm{C}_{6}$ coefficients as a function of benzene- $\mathrm{MoS}_{2}$ distance and adsorption site. We also quantify the spectral broadening of the benzene's electronic excited states due to their interaction with the surface. We find that the broadening has a similar decay law with the moleculesurface distance as the leading van der Waals interactions (i.e., $\mathrm{R}^{-6}$ ) and that the surface enhancement of dispersion interactions between benzene molecules is less than $5 \%$, but still large enough $(0.5 \mathrm{kcal} / \mathrm{mol})$ to likely play a role in the prediction of interface morphologies.
\end{abstract}




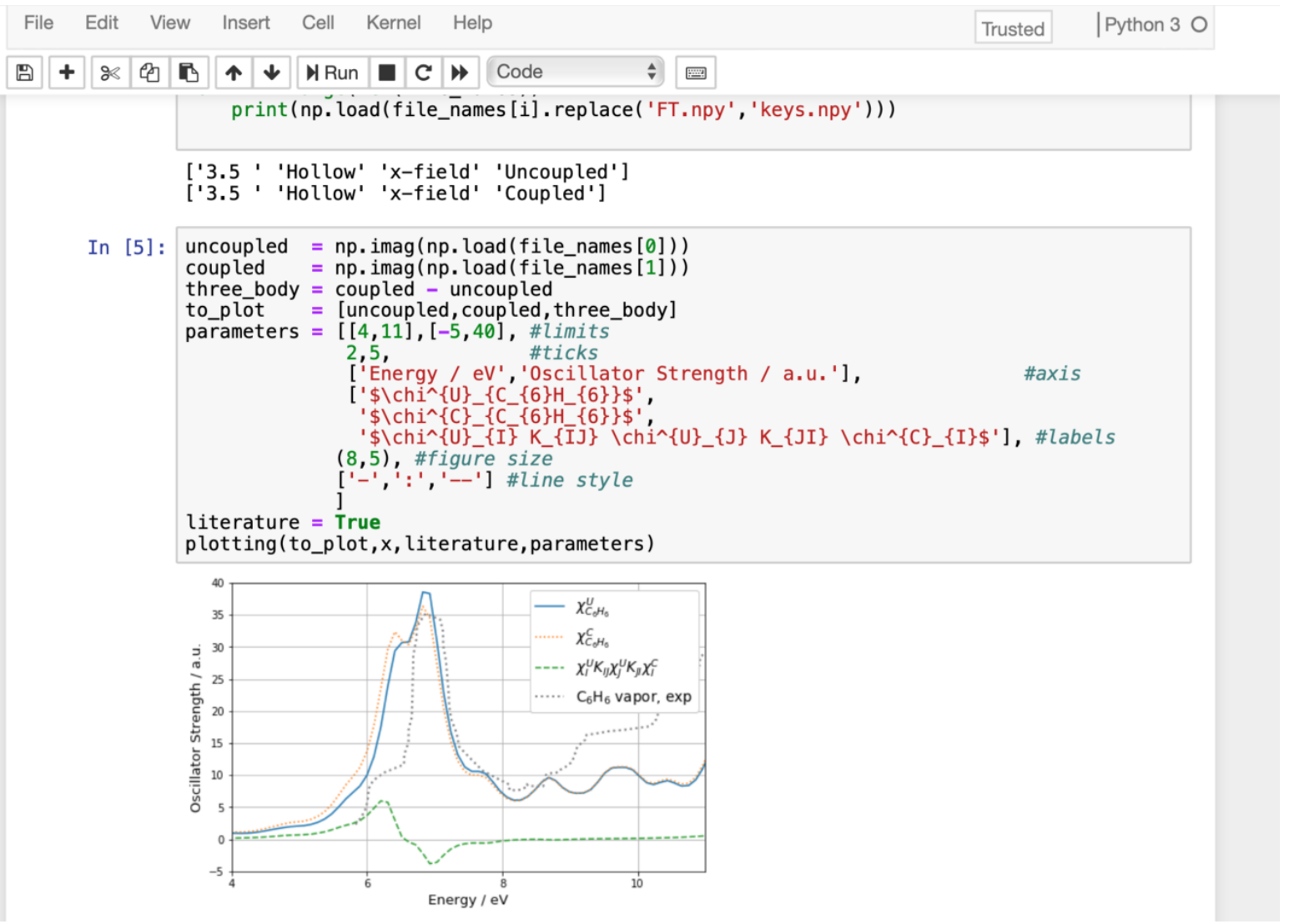

Figure 1: Jupyter Notebook with calculated values and analysis visualization. Link to source (Binder) is provided. 